\title{
Inverse Compton scattering model for gamma-ray production in $\mathrm{MeV}$ blazars
}

\author{
W. Bednarek \\ Department of Experimental Physics, University of Eódź, 90-236 Eódź, ul. Pomorska 149/153, Poland.
}

Accepted 199 Received 199; in original form 1997

\begin{abstract}
It is proposed that the spectra of so called 'MeV blazars' can be explained in terms of previously developed models of the external comptonization of accretion disk radiation provided that the structure of the inner and outer parts of the accretion disk is different. The electron acceleration is saturated by the inverse Compton losses in the inner geometrically thick disk and the outer geometrically thin disk at different maximum energies which causes the appearance of two spectral components, one strongly peaked in the $\mathrm{MeV}$ energy range and the second one of a power law type extending through the $\mathrm{GeV}$ energy range. The spectra, computed in terms of such a simple geometrical model, are in a good agreement with observations of the $\mathrm{MeV}$ blazar PKS0208-512. They are consistent with the transient appearance of a strong $\mathrm{MeV}$ peak, the power law spectrum in the EGRET energy range, and a possible cut-off at high energies.
\end{abstract}

Key words: galaxies: active - quasars: jets - radiation mechanisms: gamma-rays: galaxies:individual: PKS0208-512

\section{INTRODUCTION}

The COMPTEL telescope on the board of Compton GRO has detected a few blazars which emitted power is concentrated in the $\mathrm{MeV}$ energy range (Blom et al. 1995, Bloemen et al. 1995). Two spectral components can be clearly identified in such type of sources (e.g. PKS0208-512, Kanbach 1996, Blom et al. 1996). It has been proposed that such spectra can originate in a dense $e^{ \pm}$relativistic blob moving in a jet. In such model the $\mathrm{MeV}$ peak is caused by the blueshifted $e^{ \pm}$annihilation line (Marcowith, Henri \& Pelletier 1995, Roland \& Hermsen 1995, Böttcher \& Schlickeiser 1996, Skibo, Dermer \& Schlickeiser 1997). However observations of $3 \mathrm{C} 273$ by the OSSE telescope show that in fact the peak can be sometimes present at $\sim 0.3$ $\mathrm{MeV}$, which would rather require the redshifted $e^{ \pm}$annihilation line (McNaron-Brawn et al. 1997). Recently it has been suggested that these two emission components may be created by two different populations of electrons present in a jet, which scatter the external quasi-isotropic soft radiation (Sikora \& Madejski 1996).

It has been already noted that the spectra of $\mathrm{MeV}$ blazars could be explained by the emission of relativistic electrons which comptonize the thin disk radiation (Bednarek, Kirk, Mastichiadis 1996a,b (BKM96a,b)). However, recently observed very flat transient spectrum in the $\mathrm{GeV}$ energy range with a cut-off at a few $\mathrm{GeV}$ (Kanbach
1996) is difficult to explain with the assumption on the thin disk geometry. In this paper we show that different stages of emission of $\mathrm{MeV}$ blazars can be explained in terms of previously developed model provided that the structure of the inner and outer parts of the accretion disk is different. The electron acceleration caused, either by a shock propagating along the jet or by electric fields induced in the reconnection regions, can be saturated by inverse Compton losses. However, if the geometry of the accretion disk, surrounding central black hole, changes from the thin one (the outer disk part) to the thick one (the inner disk part) and the inner parts of the disk are relatively hotter, then the acceleration of electrons saturates at different energies during jet propagation through these radiation fields. It results in an appearance of two spectral components, the first one strongly peaked in the $\mathrm{MeV}$ energy range and the second one, of a power low type, extending through the $\mathrm{GeV}$ energy range.

\section{GAMMA-RAYS FROM SCATTERING OF PHOTONS FROM THICK AND THIN DISK}

We consider the standard external Compton scattering model for the $\gamma$-ray production in active galaxies, in which electrons accelerated in the jet lose energy on inverse Compton scattering (ICS) process by scattering the accretion disk radiation. In general, the accretion disk may be geometri- 
cally thin (as considered by e.g. Dermer, Schlickeiser \& Mastichiadis 1992, Dermer \& Schlickeiser 1993, Coppi, Kartje \& Königl 1993, BKM96a,b), or it may be geometrically thick (Melia \& Königl 1989, Bednarek 1993, Bednarek \& Kirk 1995 (BK95)). The electrons in turn may be accelerated by a relativistic shock moving in the jet (e.g. Dermer \& Schlickeiser 1993, Sikora, Begelman \& Rees 1994, Blandford \& Levinson 1995), or they move almost rectilinearly along the jet axis (Melia \& Königl 1989, Coppi, Kartje \& Königl 1993, BK95, BKM96). In this paper we consider the simpler case, assuming that the electrons are accelerated rectilinearly in the jet in a relatively small localised regions, either in electric fields induced in magnetic reconnection or by drifting along the surfaces of highly oblique shocks. Such shocks may be formed when the jet plasma meets obstacles e.g., winds of massive stars moving through the jet (Bednarek \& Protheroe 1997) or debris of close supernova explosion (Bednarek 1997). These acceleration regions are located along the axis of the jet in our model.

In the present paper we apply the model for the disk geometry considered previously by Melia \& Konigl (1989). Therefore, we assume that the outer disk, at distances greater than $r_{\text {mid }}$ from the central black hole, is geometrically thin and is characterised by the Shakura \& Sunyaev (1973) temperature profile. The inner geometrically thick disk (disk funnel), extends between $r_{\text {in }}$ and $r_{\text {mid }}$. The funnel has an opening angle $\theta$. For the outer disk we assume the Shakura \& Sunyaev temperature profile of the form

$T_{\text {out }}(r)=T_{\text {mid }}\left(r / r_{\text {mid }}\right)^{-0.75}$,

where $T_{\text {mid }}$ is the surface temperature of the disk at the distance $r_{\text {mid }}$ from the disk centre. For the temperature profile of the inner, thick disk, we assume that it has a similar form as the outer disk but with different exponent

$T_{\text {fun }}(r)=T_{\text {mid }}\left(r / r_{\text {mid }}\right)^{-\delta}$.

We discuss the cases with $\delta>0.75$, which means that the inner disk is hotter than expected in Shakura \& Sunyaev model. It is worth to note that this is consistent with the observations of the soft X-ray excess in many active galaxies, between them $3 \mathrm{C} 273$ which is also detected in $\gamma$-rays (Lichti et al. 1995). Hence the disk radiation field is defined by four parameters: $T_{m i d}, r_{m i d}, \delta$, and $\theta$. The distribution of the electric field along the jet is defined following BKM96b,

$E(z)=E_{0}\left(z / z_{0}\right)^{-\alpha}$,

where $E_{0}$ is the electric field strength at the distance $z_{0}$ along the jet, and the exponent $\alpha$ is a parameter.

The Lorentz factor of electrons in the jet, $\gamma_{e q}$, is determined by the balance between the energy gains from the rectilinear accelerator and the energy losses on ICS in the disk radiation as defined in BK95, and BKM96a,b,

$P_{I C S}\left(\gamma_{e q}(z)\right)=c e E(z)$,

where $P_{\text {ICS }}$ are the ICS losses of electrons with Lorentz factor $\gamma_{\text {eq }}$ at a distance $z, c$ is the velocity of light, and $e$ is the elementary electric charge. Bednarek, Kirk \& Mastichiadis (BKM96a,b) has shown that the jet can be separated in two regions. In the inner part, called the "radiation dominated jet", electron energy losses are strong enough that they can reach the equilibrium Lorentz factor determined by the balance between energy gains and losses. In

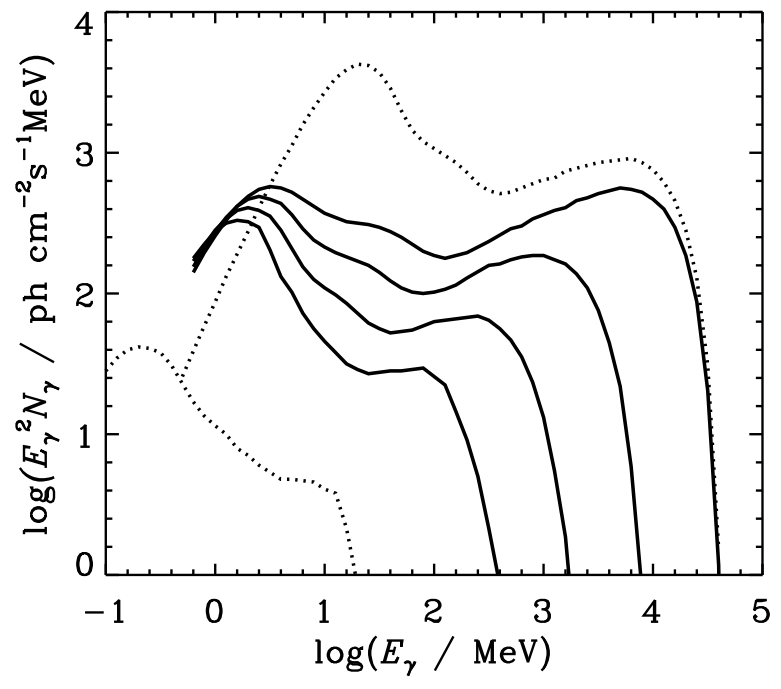

Figure 1. The ICS $\gamma$-ray spectra produced by electrons which scatter the radiation of a thick and thin accretion disk. The parameters of the disk are following: $T_{\mathrm{mid}}=5 \times 10^{4} \mathrm{~K}, r_{\mathrm{mid}}=2 \mathrm{rin}$, $r_{\text {in }}=10^{14} \mathrm{~cm}, \delta=2$, and $\theta=80^{0}$. The spectra marked by the full curves are obtained for $E_{0}=0.08 \mathrm{~V} \mathrm{~cm}^{-1}, l_{\text {rec }}=0.005$, and different values of $\alpha=0.67,1 ., 1.33,1.67$ (counting from the top). The dotted curves show the spectra for the same value of $l_{\text {rec }}$ but: $E_{0}=8 \times 10^{-3} \mathrm{~V} \mathrm{~cm}^{-1}$ (bottom dotted curves), $E_{0}=0.8 \mathrm{~V}$ $\mathrm{cm}^{-1}$ (upper dotted curve), and $\alpha=1.33$.

the outer part, called the "particle dominated jet", the ICS losses are too small and electrons are injected into the jet with the Lorentz factors corresponding to the maximum potential drop in the acceleration regions. These two regions are separated at $z_{\max }$, measured along the jet, which is given by the condition (presented in BKM96a,b),

$m_{e} c^{2} \gamma_{e q}\left(z_{\max }\right)=e l_{\operatorname{coh}} E\left(z_{\max }\right)$,

where $l_{c o h}=\beta z_{\max }$ is the coherence length for the acceleration of electrons (it can be scaled by the distance from the black hole with the scaling factor $\beta<<1), E\left(z_{\max }\right)$ is the electric field in the acceleration region (given by Eq. (3)), and $m_{e}$ is the electron rest mass. In the radiation dominated jet, the energy of electrons is directly transferred to the $\gamma$-ray photons in ICS process. In the particle dominated jet the electrons are likely to be isotropized by the random component of the magnetic field after emerging from the acceleration regions. These electrons lose energy mainly on synchrotron process contributing to the lower energy part of the blazar spectrum.

In general, electrons accelerated in the electric fields may scatter disk radiation in the Thomson or the KleinNishina case. Here we concentrate on the Thomson regime. If the scattering occurs in a narrow disk funnel, the Lorentz factor of electrons has to be limited to the values determined by the condition $\gamma_{e q}\left(3 k_{B} T / m_{e} c^{2}\right)<1$, where $k_{B}$ is the Boltzmann constant and $T$ is given by Eq 2 . If the scattering occurs above the surface of a thin disk, the condition derived in BKM96a (see Eq. (5) in that paper) has to be fulfilled.

The spectra of $\gamma$-ray photons produced in the radiation 


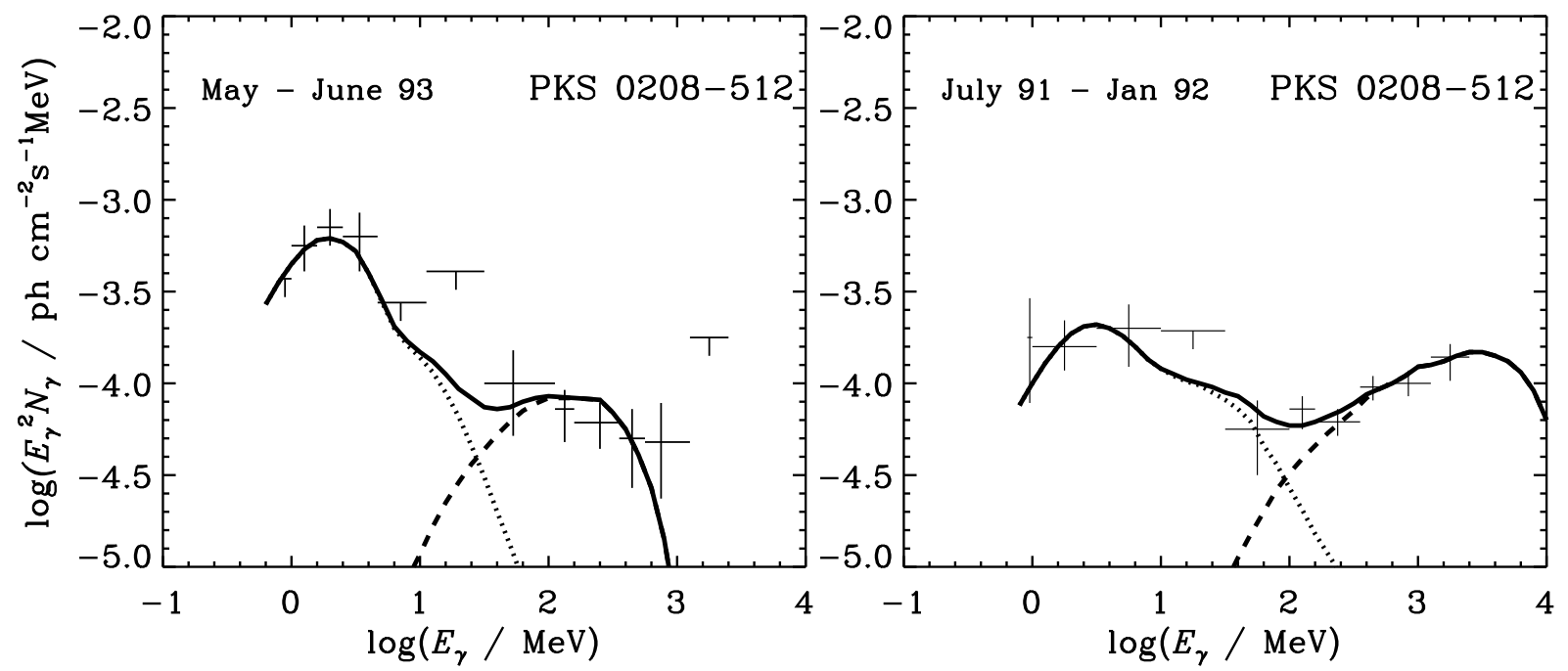

Figure 2. The spectra of $\gamma$-rays from ICS, produced by electrons propagating through radiation of the inner geometrically thick disk and the outer geometrically thin disk, are compared with the observations of MeV blazar PKS 0208-512 (Blom et al. 1996) during May - June 93 (on the left) and July 91 - Jan 92 (on the right). The differences between these spectra are only caused by the change in the distribution of the electric field profile along the jet defined by the parameter $\alpha$, which is equal to $\alpha=1.4$ for May - June 93 observations, and $\alpha=0.67$ for July 91 - Jan 92 observations. For the other parameters of the fit see text.

dominated jet are obtained by using the following formula

$\frac{d N}{d E_{\gamma} d \Omega d t}=\frac{r_{i n}}{c} \int_{z_{0}}^{z_{\max }} \frac{d N_{e}}{d \Omega d t} \frac{d N\left(\gamma_{e q}(z)\right)}{d E_{\gamma} d t} d z$,

where $d N_{e} / d \Omega d t$ is the injection rate of electrons (per $\mathrm{sr}^{-1}$ $\left.\mathrm{s}^{-1}\right), d N\left(\gamma_{e q}(z)\right) / d E_{\gamma} d t$ is the ICS $\gamma$-ray spectrum produced by an electron, with Lorentz factor $\gamma_{e q}(z)$, located at the distance $z$ along the jet. This ICS spectrum is obtained by integration of Eq. (2.33) in Blumenthal \& Gould (1970) over the disk radiation field. $z_{0}$ is taken to be equal to $r_{i n}$, and $z_{\max }$ is determined by Eq. (5).

In Fig. 1 we show an example of the $\gamma$-ray spectra calculated in terms of this model. In order to obtain these spectra we fix the disk parameters and investigate their dependence on the jet parameters. Note that the spectra show two clear components, corresponding to propagation of the jet through the radiation of the inner, geometrically thick disk (lower energy part of the spectrum) and propagation through the radiation of the outer, geometrically thin disk (higher energy part of the spectrum). The parameter $\alpha$, which describes the profile of the electric field in the jet, determines the shape of the spectrum (see full curves in Fig. 1). The location of the maximum in the power spectrum is determined by the strength of the electric field at the base of the jet (see dotted curves in Fig. 1).

\section{COMPARISON WITH THE SPECTRUM OF PKS0208-512}

PKS0208-512, at the distance corresponding to the redshift $z=1.003$, has been observed by the Compton GRO a few times (e.g. Blom et al. 1996) showing complex and variable spectrum. In some observations very strong peak was detected in $1 \div 3 \mathrm{MeV}$ energy range by the COMPTEL detector. The power law spectral component extending above $\sim 30 \mathrm{MeV}$, with the cut-off at a few $\mathrm{GeV}$, has been also detected by EGRET detector (Kanbach 1996). The spectrum is variable with the spectral index changing from $1.67 \pm 0.12$ to $2.24 \pm 0.36$ (Blom et al. 1996). We interpret this complex spectral shape in terms of our simple one component jet model and Melia \& Königl (1989) geometrical disk model. It is assumed that the disk parameters has not been changed between these two observations. The disk is defined by the inner disk radius $r_{i n}=10^{14} \mathrm{~cm}$, the radius of the thick disk $r_{\text {mid }}=2 r_{i n}$, its temperature $T_{m i d}=5 \times 10^{4} \mathrm{~K}$ at $r_{m i d}$, the exponent $\delta=2$, and the opening angle of the thick disk $\beta=80^{\circ}$. The fits to the spectra of PKS 0208-512 are shown in Fig. 2 during different observations (Blom et al. 1996). The spectra are obtained for the following parameters of the jet: $E_{0}=0.08 \mathrm{~V} \mathrm{~cm}^{-1}$ at the base of the jet $z_{0}=r_{i n}$, and $l_{\text {rec }}=0.005$. We assumed that the injection rate of electrons is uniform along the jet. This might be motivated by the fact that the flux of the electrons in the jet should be conserved.

The change in the spectra, during two observations of PKS 0208-512, is caused only by changing the distribution of the electric field along the jet, defined by the parameter $\alpha$ (see Eq. 3). Its value is equal to 1.4, for the observations during May - June 93, and to 0.67, for the observations during July 91 - Jan 92 . The change in these spectra corresponds to different equilibrium Lorentz factors of electrons during propagation in the electric field of the jet. In Fig. 3 we present the values of $\gamma_{\mathrm{eq}}$ as a function of the distance $z$ along the jet, for two cases discussed above. Note the difference in extraction of the electric field energy by electrons propagating through the radiation fields of thick and thin disk in both cases. This is the reason of anticorrelation of relative power emitted at the high energy (above $\sim 100 \mathrm{MeV}$ ) and the low energy parts of the spectrum. In such model the cut-off in the spectrum, observed at around a few GeV (Kanbach 1996), is caused by inefficient acceleration of electrons in the electric 
field of the particle dominated jet. This interpretation is different from another possible explanation of such a feature as being due to absorption of high energy $\gamma$-rays in the soft radiation (e.g. Bednarek 1993, Sikora, Begelman \& Rees 1994, BK95, Blandford \& Levinson 1995, Pohl 1996).

\section{CONCLUSION}

We propose a simple geometric explanation as responsible for the formation of the two component $\gamma$-ray spectrum in $\mathrm{MeV}$ blazars. It is shown that in terms of the standard external comptonization model of the disk radiation, the two components in the $\gamma$-ray spectrum can be explained if the disk geometry changes from the geometrically thin one, in the outer part, to the geometrically thick one, in the inner part. Computed $\gamma$-ray spectra can well describe the complex shape of the most prominent MeV blazar PKS0208-512 (Fig. 2). The model is able to explain the relative changes in the strength of the soft $\mathrm{MeV}$ component and hard component above $\sim 30 \mathrm{MeV}$. It is also consistent with the high energy cut-off in the $\gamma$-ray energy range. Since the lower $\gamma$-ray emission is produced closer to the central engine, it is likely that the $\mathrm{GeV}$ emission, produced further along the jet, is delayed in respect to the $\mathrm{MeV}$ emission. This model can also simply explain the appearance of a bump in the blazar spectra at energies below $\sim 0.511 \mathrm{MeV}$ (the case of 3 C273, McNaron-Brawn et al. 1997), which can not be explained by the $e^{ \pm}$annihilation in the jet model. The peak in the $\gamma$-ray power spectrum, predicted by our model, is only determined by the balance between energy gains and energy losses of electrons propagating inside the thick disk (see Fig. 1). Therefore, it may appear in principle, at arbitrary energy.

It is assumed in this paper that the electrons are accelerated rectilinearly as discussed originally by Bednarek \& Kirk (BK95). However, we expect that similar two component spectra should appear if electrons, accelerated by a relativistic shock propagating along the jet axis, scatter radiation of the inner and outer disk. The acceleration of electrons can be also saturated by the ICS losses and the maximum energy of electrons is determined by the balance between acceleration efficiency and energy losses. Only the geometry of the ICS scattering process by electrons distributed isotropically in the blob frame becomes more complicated as shown by e.g. Dermer \& Schlickeiser (1993).

\section{ACKNOWLEDGEMENTS}

I thank Dr H. Blom for providing me with the data on the gamma-ray observations of PKS 0208-512 by the COMPTEL and EGRET detectors on Compton GammaRay Observatory, the referee for comments, the editor for suggestion, and Dr M. Bałucińska-Church for reading the manuscript.

\section{REFERENCES}

Bednarek, W., 1993, ApJ, 402, L29

Bednarek, W., 1997, in Proc. Frascati Workshop, eds. F. Giovannelli \& L. Sabau-Graziati, Memorie S.A.It. (Italy), submitted

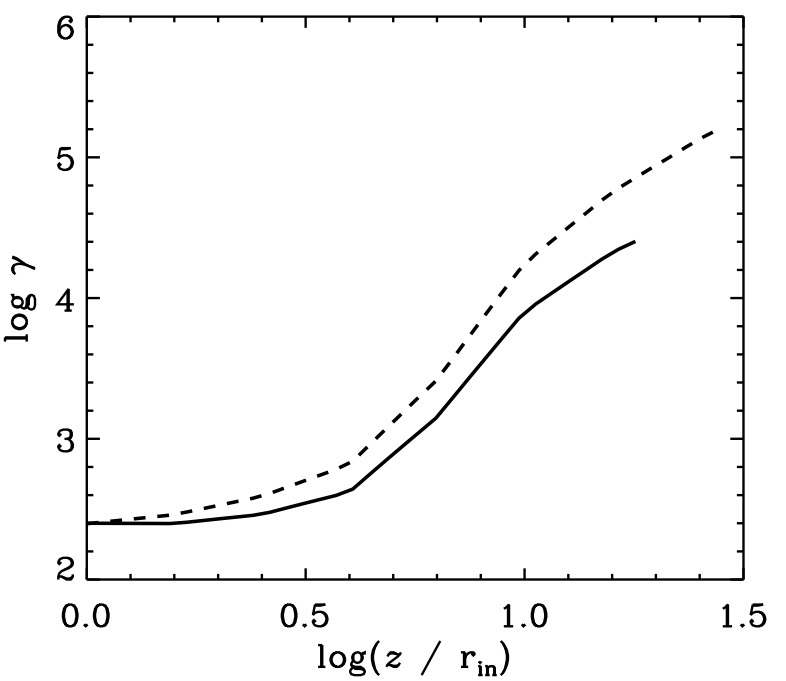

Figure 3. The equilibrium Lorentz factor of electrons as a function of the propagation distance along the jet for which the $\gamma$-ray spectra presented in Figs. 2 are obtained and compared with May - June 93 observations (full curve) and July 91 - Jan 92 observations (dashed curve).

Bednarek, W., Kirk, J.G., 1995, A\&A, 294, 366 (BK95)

Bednarek, W., Kirk, J.G., Mastichiadis, A., 1996, A\&A, 307, L17 (BKM96a)

Bednarek, W., Kirk, J.G., Mastichiadis, A., 1996, A\&AS, 120, 571 (BKM96b)

Bednarek, W., Protheroe, R.J., 1997, MNRAS, 287, L9

Bertsch, D.L. et al. 1993, ApJ, 405, L21

Blandford, R.D., Levinson, A., 1995, ApJ, 441, 79.

Bloemen, H. et al., 1995, A\&A, 293, L1

Blom, H. et al., 1995, A\&A, 298, L33

Blom, H. et al., 1996, A\&AS, 120, 507

Blumenthal, G.R., Gould, R.J., 1970, Rev.Mod.Phys., 42, 237

Böttcher, M., Schlickeiser, R., 1996, A\&AS, 120, 575

Coppi, P.S., Kartje, J.F., Königl, A., 1993, Proc. Compton Gamma-Ray Observatory, eds. M. Friedlander, N. Gehrels \& D.J. Macomb (New York: AIP), AIP v. 280, 559

Dermer, C.D., Schlickeiser, R., 1993, ApJ, 416, 458

Dermer, C.D., Schlickeiser, R., Mastichiadis, A., 1992, A\&A, 256, L27

Kanbach. G. 1996, Proc. Heidelberg Workshop on GAMMA-RAY EMITTING N, eds. J.G. Kirk, M. Camenzind, C. von Montigny, S. Wagner, Heidelberg (MPI H -V37 - 1996), p. 1

Lichti, G. et al., 1995, A\&A, 298, 711

Marcowith, A., Henri, G., Pelletier, G., 1995, MNRAS, 277, 681

McNaron-Brawn, K. Johnson, W.N., Dermer, C.D., Kurfess, J.D., 1997, ApJ, 474, L85

Melia, F. \& Königl, A., 1989, ApJ, 340, 162

Pohl, M. 1996, Proc. Heidelberg Workshop on GAMMA-RAY EMITTING N, eds. J.G. Kirk, M. Camenzind, C. von Montigny, S. Wagner, Heidelberg (MPI H -V37 - 1996), p. 23

Roland, J., Hermsen, W., 1995, A\&A, 262, 26

Shakura, N.I., Sunyaev, R.A., 1973, A\&A, 24, 337

Sikora, M., Begelman, M.C., Rees, M.J., 1994, ApJ, 421, 153.

Sikora, M., Madejski, G., 1996, Proc. Heidelberg Workshop on GAMMA-RAY EMITTING N, eds. J.G. Kirk, M. Camenzind, C. von Montigny, S. Wagner, Heidelberg (MPI H -V37 - 1996), p. 153

Skibo, J.G., Dermer, C.D., Schlickeiser, R., 1997, ApJ, in press 